\title{
Spatial variation of the cooling lines in the reflection nebula NGC 7023 ${ }^{\star}$
}

\author{
J. Bernard-Salas ${ }^{1,2}$, E. Habart ${ }^{2}$, M. Köhler ${ }^{2}$, A. Abergel ${ }^{2}$, H. Arab ${ }^{2,3}$, V. Lebouteiller ${ }^{4}$, C. Pinto ${ }^{5}$, \\ M. H. D. van der Wiel ${ }^{6}$, G. J. White ${ }^{1,7}$, and M. Hoffmann ${ }^{2,8}$
}

\author{
1 Department of Physical Sciences, The Open University, MK7 6AA, Milton Keynes, UK \\ e-mail: jeronimo.bernard-salas@open.ac.uk \\ 2 Institut d'Astrophysique Spatiale, Paris-Sud 11, 91405 Orsay, France \\ 3 Space Telescope Science Institute, 3700 San Martin Drive, Baltimore, MD 21218, USA \\ ${ }^{4}$ Laboratoire AIM, CEA/DSM-CNRS-Universite Paris Diderot, DAPNIA/Service d'Astrophysique, Saclay, France \\ 5 Laboratoire d'Astrophysique de Marseille, CNRS/INSU Université de Provence, 13388 Marseille, France \\ ${ }^{6}$ Institute for Space Imaging Science, Department of Physics \& Astronomy, University of Lethbridge, Lethbridge AB T1K 3M4, \\ Canada \\ 7 Space Science \& Technology Division, The Rutherford Appleton Laboratory, Chilton, Didcot OX11 0NL, UK \\ 8 Wolfson Brain Imaging Centre, Addenbrookeś Hospital, University of Cambridge, Cambridge CB2 0QQ, UK
}

Received 24 February 2014 / Accepted 4 December 2014

\section{ABSTRACT}

\begin{abstract}
Context. The north-west photo-dissociation region (PDR) in the reflection nebula NGC 7023 displays a complex structure. Filamentlike condensations at the edge of the cloud can be traced via the emission of the main cooling lines, offering a great opportunity to study the link between the morphology and energetics of these regions.

Aims. We study the spatial variation of the far-infrared fine-structure lines of [C II] (158 $\mu \mathrm{m})$ and [O I] (63 and $145 \mu \mathrm{m})$. These lines trace the local gas conditions across the PDR. We also compare their emission with molecular tracers including rotational and rovibrational lines of $\mathrm{H}_{2}$ and high-rotational lines of $\mathrm{CO}$.

Methods. We used observations from the Herschel/PACS instrument to map the spatial distribution of these fine-structure lines. The observed region covers a square area of about $110^{\prime \prime} \times 110^{\prime \prime}$ with an angular resolution that varies from $4^{\prime \prime}$ to $11^{\prime \prime}$. We compared this emission with ground-based and Spitzer observations of $\mathrm{H}_{2}$ lines, Herschel/SPIRE observations of CO lines, and Spitzer/IRAC 3.6 $\mu \mathrm{m}$ images that trace the emission of polycyclic aromatic hydrocarbons. We used a PDR code to model the [O I] $145 \mu \mathrm{m}$ line and infer the physical conditions in the region.

Results. The [C II] $(158 \mu \mathrm{m})$ and [OI] $(63$ and $145 \mu \mathrm{m})$ lines arise from the warm cloud surface where the PDR is located and the gas is warm, cooling the region. We find that although the relative contribution to the cooling budget over the observed region is dominated by [OI] $63 \mu \mathrm{m}(>30 \%), \mathrm{H}_{2}$ contributes significantly in the PDR ( 35\%), as does [C II] $158 \mu \mathrm{m}$ outside the PDR (30\%). Other species contribute little to the cooling ([O I] $145 \mu \mathrm{m} \mathrm{9 \% ,} \mathrm{and} \mathrm{CO} \mathrm{4 \% ).} \mathrm{Enhanced} \mathrm{emission} \mathrm{of} \mathrm{these} \mathrm{far-infrared} \mathrm{atomic} \mathrm{lines}$ trace the presence of condensations, where high-excitation $\mathrm{CO}$ rotational lines and dust emission in the submillimetre are detected as well. The [O I] maps resolve these condensations into two structures and show that the peak of [O I] is slightly displaced from the molecular $\mathrm{H}_{2}$ emission. The size of these structures is about $8^{\prime \prime}(0.015 \mathrm{pc})$ and in surface cover about $9 \%$ of the PDR emission. We have tested whether the density profile and peak densities that were derived in previous studies to model the dust and molecular emission can predict the [O I] $145 \mu \mathrm{m}$ emission. We find that the model with a peak density of $10^{6} \mathrm{~cm}^{-3}$, and $2 \times 10^{4-5} \mathrm{~cm}^{-3}$ in the oxygen emitting region predicts an [O I] $145 \mu \mathrm{m}$ line that is only $30 \%$ lower than the observed emission. Finally, we did not detect emission from [N II] $122 \mu \mathrm{m}$, suggesting that the cavity is mostly filled with non-ionised gas.
\end{abstract}

Key words. infrared: general - H II regions - photon-dominated region (PDR) - ISM: individual objects: NCG 7023 ISM: lines and bands

\section{Introduction}

NGC 7023 is a bright and well-known reflection nebula. Although it is a relatively nearby object, its reported distance varies significantly in the literature. Using HIPPARCos parallaxes, van den Ancker et al. (1997) and later on van Leeuwen (2007) measured a distance of $430_{-90}^{+160} \mathrm{pc}$ and $520 \pm 180 \mathrm{pc}$, respectively, and Benisty et al. (2013) estimated a distance of $320 \pm 51 \mathrm{pc}$ based on revised orbital parameters and

\footnotetext{
* Herschel is an ESA space observatory with science instruments provided by European-led Principal investigator consortia and with important participation from NASA.
}

astrometry. In this paper we adopt the distance given by van den Ancker et al. (1997) (430 pc), which is almost the average of the values given by van Leeuwen (2007) and Benisty et al. (2013).

The reflection nebula is excited by the Herbig Be binary star system HD 200775 (Alecian et al. 2008), which is immersed in the cavity of the molecular cloud (Fuente et al. 1992). The overall morphology of NGC 7023 is driven by the action of the ionising stars, where the stellar outflow, now inactive, creates a biconical cavity in the shape of a butterfly of about of $1.5 \mathrm{pc} \times 0.8 \mathrm{pc}$ in size (Köhler et al. 2014). The stars illuminate the walls of the cavity in an almost edge-on orientation. At the edge of 
these walls, three photo-dissociation regions (PDRs) are present (Fuente \& Martín-Pintado 1997), which are located at 40" northwest (NW), 70" south-west (SW), and 170" east of the stars. The NW PDR is the brightest and is oriented almost edge-on to the observer. Polarization observations in the near-infrared (Sellgren et al. 1992) and vibrational $\mathrm{H}_{2}$ (Lemaire et al. 1996) of the NW PDR reveal a filamentary morphology. Witt et al. (2006) found that this morphology is the result of several condensations that are superimposed along the line of sight, whose surfaces are directly illuminated by the star. The incident radiation field, in units of the interstellar far-ultraviolet radiation field estimated by Habing $^{1}$, is $G=2.6 \times 10^{3}$ (Chokshi et al. 1988; Pilleri et al. 2012). However, higher values also having been reported in the literature (Fuente et al. 1999). Deriving the incident radiation field relies on several assumptions on the geometry and spectral type of the star. For the rest of the paper, we assume the value of $G=2.6 \times 10^{3}$ at the PDR front, as in Chokshi et al. (1988) and Pilleri et al. (2012).

NGC 7023 has been the subject of many studies in the literature, including several spectroscopic studies in the mid- and far-infrared (FIR). The mid- and far-IR spectral regions contain many important cooling lines and hold the fingerprints of the dust emission. Fuente et al. (2000) presented observations of NGC 7023 from the Infrared Space Observatory (ISO) with the short and long wavelength spectrometers (SWS and LWS) and differentiated between three regions: 1) the star and the cavity formed by the star, which is filled with a low-density gas; 2) the edges of the cavity that defines the PDRs; and 3) the molecular gas. Based on their analysis of the atomic and molecular lines ([O I]63 $\left.\mu \mathrm{m},[\mathrm{OI}] 145 \mu \mathrm{m},[\mathrm{C} \mathrm{II}] 158 \mu \mathrm{m}, \mathrm{H}_{2}\right)$, they found that the NW and SW PDRs have similar excitation conditions, with filaments with a high density of $n \sim 10^{6} \mathrm{~cm}^{-3}$ and a lower density of $n \sim 10^{4} \mathrm{~cm}^{-3}$ in the region between filaments. Werner et al. (2004) and, later on, Sellgren et al. (2007) studied the variation of the dust features in the mid-IR spectrum of NGC 7023 using the Spitzer Infrared Spectrograph (IRS) and Multiband Imaging Photometers (MIPS) instruments. They observed variations in the NW and SW PDRs of the intensity, widths, and central wavelength of the spectral features that arise from polycyclic aromatic hydrocarbons (PAHs) as a function of the distance to the ionising source (HD 200775). Witt et al. (2006) found a good correlation between the optical emission from the dust, as traced by the $R$ band $(658 \mathrm{~nm})$, with that of rovibrational $\mathrm{H}_{2}$. Using Spitzer data, Fleming et al. (2010) studied the $\mathrm{H}_{2}$ and PAHs emission in the PDRs of three reflection nebulae, including NGC 7023. They showed evidence for PAH de-hydrogenation, which they concluded to be suggestive of $\mathrm{H}_{2}$ formation on PAHs.

Recently, NGC 7023 has been the target of several programs using the Herschel Space Observatory (Pilbratt et al. 2010), vastly improved the spatial resolution of previous FIR studies. Abergel et al. (2010) combined Spitzer and Herschel maps to study the spatial variations of the dust properties in the eastern PDR. Using a radiative transfer code, they were able to reproduce the different spatial variations of PAHs, very small grains (VSGs), and large dust grain emission. Joblin et al. (2010) used the high spectral resolution provided by the HIFI instrument to study the $[\mathrm{C}$ II $] 158 \mu \mathrm{m}$ line at several positions in the nebula, including a central position in the NW PDR. They found that the emission of $[\mathrm{C}$ II $] 158 \mu \mathrm{m}$ line and PAHs arises from the transition region between the atomic and molecular gas.

\footnotetext{
1 The Habing field corresponds to $1.6 \times 10^{-3} \mathrm{erg} \mathrm{s}^{-1} \mathrm{~cm}^{-2}$ when integrated between 91.2 and $240 \mathrm{~nm}$ (Habing 1968).
}

Okada et al. (2013) studied the photoelectric efficiency from PAHs in a sample of six PDRs, including the east and NW PDRs of NGC 7023. Using Spitzer and Herschel/PACS observations, they evaluated the photo-electric effect from PAHs and the cooling [O I]63 and $145 \mu \mathrm{m}$, and [C II] $158 \mu \mathrm{m}$ lines in three regions (cavity, interface, and molecular region) and found that regions with a high fraction of ionised PAHs have a lower heating efficiency.

In this paper we present observations from the Photoconductor Array Camera and Spectrometer (PACS, Poglitsch et al. 2010) of the [OI]63 $\mu \mathrm{m},[\mathrm{OI}] 145 \mu \mathrm{m}$, and $[\mathrm{C}$ II $] 158 \mu \mathrm{m}$ lines in the NW PDR of NGC 7023. These observations allow us to resolve in detail the global shape of the PDR, although substructures such as the filaments observed at higher spatial resolution (e.g., Lemaire et al. 1996; Fuente et al. 1996) are unresolved. This study complements a recent SPIRE spectroscopic study of the molecular gas and dust emission by our group (Köhler et al. 2014). This paper is organised as follows: the observations and data reduction are described in Sect. 2. In Sect. 3 we discuss the spatial morphology of the lines, compare them with the emission of $\mathrm{CO}$ and $\mathrm{H}_{2}$, and describe the correlation between the different line ratios as a function of the distance to the ionising source. In Sect. 4 we analyse the cooling budget and the morphology of the main cooling lines. In Sect. 5 the results of the modelling are described. The conclusions are summarised in the final section.

\section{Observations and data reduction}

The observations were carried out during the science demonstration phase (SDP) of Herschel on 7 January 2010 and are part of the "Evolution of the Interstellar Medium" guaranteedtime key project (observation ID $=1342191152$, Abergel et al. 2010). The observations were taken using the PACS instrument in the - now decommissioned - wavelength-switching mode. Four fine-structure lines were observed: [C II] at $158 \mu \mathrm{m},[\mathrm{OI}]$ at 63 and $145 \mu \mathrm{m}$, and [N II] at $122 \mu \mathrm{m}$. The observations were centred on the NW PDR, with RA and Dec coordinates of $315.375^{\circ}$ and $60.178^{\circ}$, respectively (J2000).

The observing strategy and reduction methods follow that of our earlier paper on the Orion Bar (Bernard-Salas et al. 2012). We thus summarise the main steps here and refer to the above paper for details.

To trace the NW PDR, we performed a $3 \times 3$ raster map. The configuration at the time of the observation is shown in Fig. 1, where the raster map at the epoch of observation is overlaid on top of an $\mathrm{H}_{2}$ map of the region made with the Canada-FranceHawaii Telescope (CFHT). An additional map centred on the eastern PDR (not shown in the figure) will be presented in a future paper. A minimum exposure configuration of one cycle and repetition per line was performed. Raster and point line steps of 23.5" were chosen and result in Nyquist sampling for the lines in the red channel ([C II $] 158 \mu \mathrm{m},[\mathrm{OI}] 145 \mu \mathrm{m},[\mathrm{N} \mathrm{II}] 122 \mu \mathrm{m})$. The purpose of the wavelength-switching mode is to cancel out the background by determining a differential line profile. This means that observations at an off-position are not needed to remove the background in such mode. The present observations of NGC 7023 were deemed to have calibration value by the Herschel Science Center, and it was decided to obtain an additional set of observations at the centre position so that the PDR would be observed with a higher signal-to-noise ratio $(\mathrm{S} / \mathrm{N})$. This was achieved by including an off-observation in the astronomical observation request (AOR), to be performed after three raster positions and with zero offset relative to the centre of the map. 


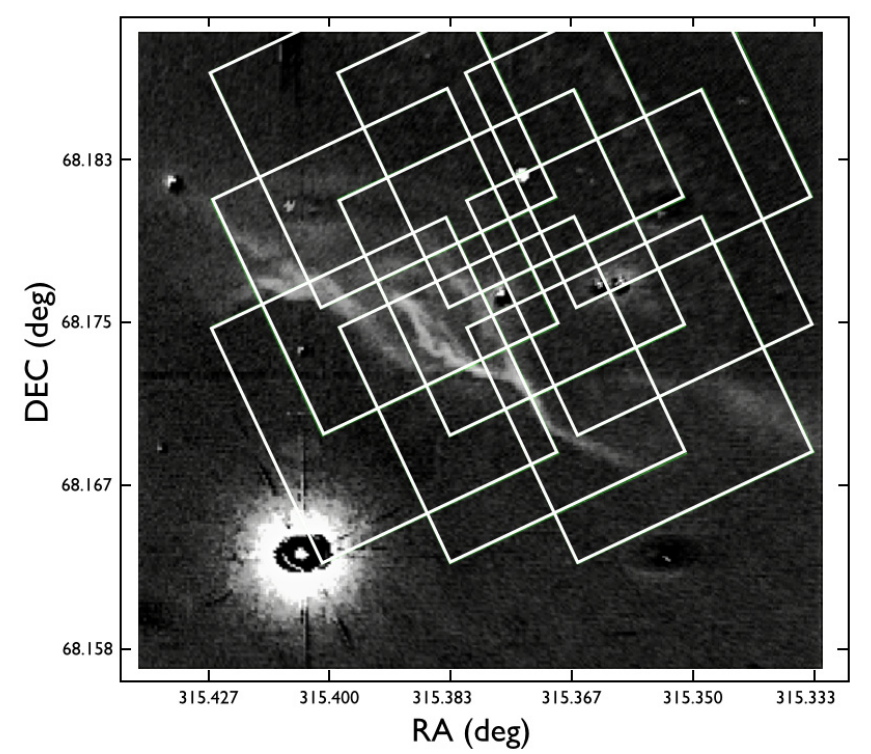

Fig. 1. Overlay of the PACS field of view on a ground-based $\mathrm{H}_{2}$ 1-0 S(1) map at $2.12 \mu \mathrm{m}$ (Lemaire et al. 1996). A $3 \times 3$ raster map was performed (see Sect. 2). The map covers an area of approximately $110^{\prime \prime} \times 110^{\prime \prime}$ and is centred on the NW PDR. The emission from the ionising stars (HD 200775), in the bottom left corner of the figure, is saturated. RA and Dec coordinates are in degrees (J2000).

This resulted in the centre of the map being observed a total of three times, with the redundancy resulting in a higher $\mathrm{S} / \mathrm{N}$ for that central position, which is centred on the PDR.

The data were processed using version 6.0.3 of the reduction and analysis package HIPE (Ott 2010). Each raster position, or footprint, consists of $5 \times 5$ spectral pixels (called spaxels). HIPE produces a data cube for each footprint, which contains a spectrum for each spaxel. Starting from level 1, the cubes were further processed, using proprietary tools, to correct for minor drifting effects and flux misalignments between scans. At this point the cubes were exported into the software PACSman (Lebouteiller et al. 2012) to measure the line fluxes by fitting a Gaussian and to create the final map. The uncertainties in the line fluxes are small and on average amount to less than $5 \%$ for the $[\mathrm{CII}]$ and $[\mathrm{OI}]$ lines. The relative flux accuracy between spaxels is $10 \%^{2}$, and for the remaining of the paper we have adopted this value or the uncertainty from the fit given by PACSman, whichever is higher. Note that we did not detect the $[\mathrm{N} \mathrm{II}] 122 \mu \mathrm{m}$ line at any position in the map. The final integrated intensity maps of the [O I]63 $\mu \mathrm{m}$, [O I] $145 \mu \mathrm{m}$, and [C II] $158 \mu \mathrm{m}$ lines are shown in Fig. 2.

We compared our measurements of the [C II] line with the fluxes measured by Joblin et al. (2010) using the HIFI instrument, and the ISO/LWS measurements by Fuente et al. (2000), all compared to the largest beam. The position used for comparison $\left(\mathrm{RA}=315.385^{\circ}, \mathrm{Dec}=68.1743^{\circ}\right)$ is located in the clump of enhanced emission and is marked at the top right panel of Fig. 2 with a plus symbol. All measurements agree within $\sim 10 \%$. Okada et al. (2013) observed the same lines in the NW PDR. Their observations consist of a single pointing, while our observations perform a detailed mapping of the region to show the morphology of these lines, providing a complete coverage of the region and allowing for better calibration.

\footnotetext{
2 From the PACS spectroscopy performance and calibration manual. This can be found at http://herschel.esac.esa.int/twiki/ bin/view/Public/PacsCalibrationWeb
}

We compared our fluxes with with those of Okada et al. (2013) in the cavity, interface, and molecular region. These three positions are marked with squares in the top right panel of Fig. 2. All values agree within $29 \%$, where the [C II] $158 \mu \mathrm{m}$ and [OI]145 $\mu \mathrm{m}$ fluxes in the interface, and the $[\mathrm{OI}] 63 \mu \mathrm{m}$ flux in the molecular region agree within $5 \%$. The uncertainty assumed by Okada et al. (2013) is of $\sim 30 \%$, while in our work it is typically $<10 \%$, and thus both values are consistent within the uncertainties.

In addition to the atomic lines presented in this paper, we compare in Sect. 4 their emission with other relevant cooling species of $\mathrm{CO}, \mathrm{H}_{2} \mathrm{O}, \mathrm{CH}^{+}$, and $\mathrm{C}^{0}$. In particular, we use the CO $4<J_{\mathrm{u}}<13$ transitions in the SPIRE range, and the rotational lines of $\mathrm{H}_{2}$ 0-0 S(1), S(2), and $\mathrm{S}(3)$ at 17.0, 12.3, and $9.7 \mu \mathrm{m}$ from the Spitzer data. We also use the 360 and $609 \mu \mathrm{m}$ lines of $C^{0}$ (SPIRE). Since we did not detect any $\mathrm{CH}^{+}$and $\mathrm{H}_{2} \mathrm{O}$ lines in the SPIRE range, we derived an upper limit to the transitions of these species that were detected in the Orion Bar (Bernard-Salas et al. 2012). These observations were taken from Pilleri et al. (2012) for the $\mathrm{H}_{2}$ lines and from Köhler et al. (2014) for the SPIRE lines.

\section{Spatial distribution}

\subsection{Cooling lines}

The three top panels in Fig. 2 show the observed distributions of the [OI]63 and $145 \mu \mathrm{m}$, and [C II] $158 \mu \mathrm{m}$ lines. The exciting source, the binary system HD $200775\left(\mathrm{RA}=315.404^{\circ}\right.$, Dec $\left.=68.1633^{\circ}\right)$, illuminates the PDR from the lower left side of the figure (just outside the maps, see also Fig. 3). For each panel, the beams are shown in the lower left corner and represent sizes of $4.5^{\prime \prime}, 8.8^{\prime \prime}$, and $11^{\prime \prime}$ from left to right. In this scale, and adopting a distance of $430 \mathrm{pc}$ to NGC 7023, 10" corresponds to a physical scale of $0.02 \mathrm{pc}$.

The PDR is detected in the three lines. The emission of these lines arises from the surface of the clouds, where the gas is warm ( 700-70 K, Fuente et al. 1999; Fleming et al. 2010; Köhler et al. 2014). There is also evidence for condensations of enhanced emission within the PDR, where both [O I]63 and $145 \mu \mathrm{m}$ line maps can resolve two structures (red clumps in the figure). This is the first time in NGC 7023 that such structures have been revealed from the emission of the $[\mathrm{C} \mathrm{III}] 158 \mu \mathrm{m}$ and [O I]63 and $145 \mu \mathrm{m}$ lines within the PDR. These structures or knots are directly illuminated by the star and coincide with the presence of highly excited $\mathrm{CO}$ lines and dust emission (see next section). These clumps are better delineated in the [O I]145 $\mu \mathrm{m}$ map (Fig. 2), which reveals two distinct clumps, and a possibly third fainter clump. The size of the main structures is about $8^{\prime \prime}(0.015 \mathrm{pc}$ at the distance to NGC 7023). While it is possible that these clumps may not be yet be resolved in our observations, we find that these clumps cover $9 \%$ of the PDR surface area, where in this case we consider the PDR area to be any [O I] $145 \mu \mathrm{m}$ flux greater than $0.9 \times 10^{-7} \mathrm{~W} \mathrm{~m}^{-2} \mathrm{sr}^{-1}$ (red, green, and blue colours in the upper middle panel of Fig. 2).

The PDR is well delineated in the [O I] $145 \mu \mathrm{m}$ line (Fig. 2). Farther away from HD 200775, the emission of [OI] $145 \mu \mathrm{m}$ drops to zero. The spatial distribution of the [OI]63 $\mu \mathrm{m}$ line is similar, although the emission is more extended than that of the $[\mathrm{OI}] 145 \mu \mathrm{m}$ line. This is expected as the $[\mathrm{OI}] 63 \mu \mathrm{m}$ line becomes optically thick at lower column densities than the [OI]145 $\mu \mathrm{m}$ line, and once saturated, its emission will be broader (Caux et al. 1999). The [C II] $158 \mu \mathrm{m}$ emission is even more extended because it is easily 


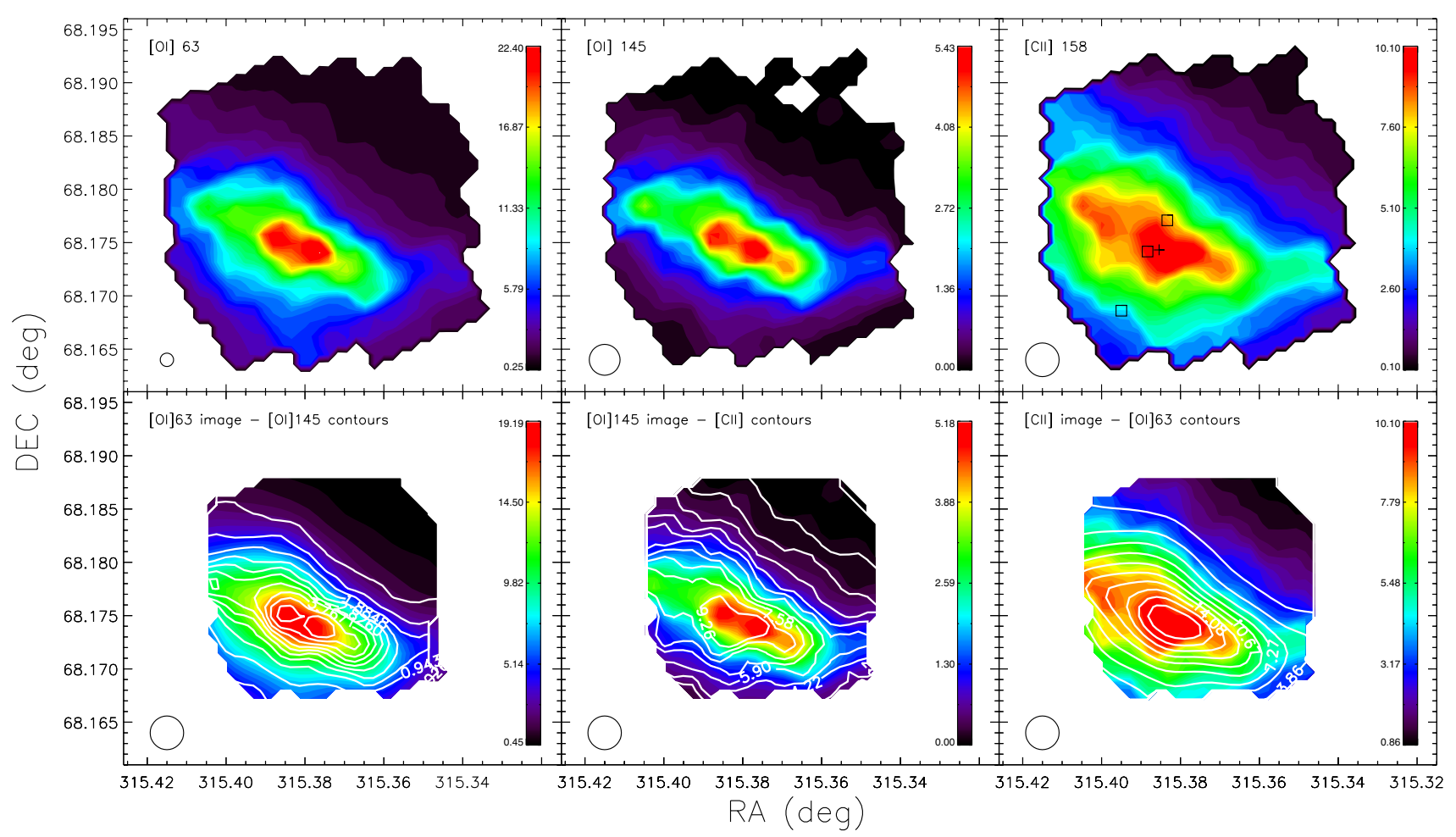

Fig. 2. Upper three panels: observed images for the [O I]63 $\mu \mathrm{m}$, [O I] $145 \mu \mathrm{m}$, and [C II] $158 \mu \mathrm{m}$ lines. Lower panels: combination of lines with contour maps over-plotted in white. The bottom images have been convolved to the $158 \mu \mathrm{m}$ (largest) beam, with the beam size illustrated in the bottom left of each panel. The squares and plus symbols in the upper right panel indicate the positions used to compare the [C II] $158 \mu \mathrm{m}$ flux with previous measurements in the literature (see Sect. 2). All maps are in flux units of $10^{-7} \mathrm{~W} \mathrm{~m}^{-2} \mathrm{sr}^{-1}$. The eight highest contours are at $100,91,82$, $74,65,56,47$, and $38 \%$ of the peak emission.

excited. This was also seen in a similar study by our group on the Orion Bar (Bernard-Salas et al. 2012). The figure also shows that the morphology of the [C II] $158 \mu \mathrm{m}$ line is complex and structured. Using Herschel/HIFI high-resolution spectra of the [C II] $158 \mu \mathrm{m}$ line, Joblin et al. (2010) and Berné et al. (in prep.) detected different components with a wide distribution of velocities, indicating that in addition to the main filaments, other regions are contributing to the [C II] emission.

In the lower three panels of Fig. 2 we plot different combinations of lines and their contours convolved to the largest beam size $\left(11^{\prime \prime}\right)$. Both of the [O I] lines correlate spatially very well with each other (lower left panel). The middle bottom panel shows that the [C II] emission also delineates the PDR, but with a more complex structure than the [OI] $145 \mu \mathrm{m}$ line. The peak emission is slightly displaced and broader in the [C II] line than the $[\mathrm{O} \mathrm{I}] 145 \mu \mathrm{m}$. This is better seen in the last panel, where the concentric contours of the [OI]63 $\mu$ m line contrasts with the more irregular emission of [C II], including a peak of emission with a heart-shaped structure.

Finally, as mentioned in the previous section, no [N II] line at $122 \mu \mathrm{m}$ was detected in the PACS map. The $205 \mu \mathrm{m}$ line was not detected in the SPIRE maps either (Köhler et al. 2014). Using HIFI observations that include the ionising stars, the Herschel Warm and Dense ISM program (WADI, Ossenkopf et al. 2011) only detects ionised emission from a shell close to the stars (priv. comm.), an area just outside our observed map. This could indicate that the gas in the cavity near the PDR is mostly neutral and not ionised and might explain why we did not detect the $122 \mu \mathrm{m}$ line since our map does not include this region close to the ionising stars. Alternatively, if the density is too low in the cavity (Fuente et al. 2000), its emission will be too weak to be detected in our observations. We have derived an upper limit to the flux for this line of $1.1 \times 10^{-7} \mathrm{~W} \mathrm{~m}^{-2} \mathrm{sr}^{-1}$.

\subsection{Comparison with $\mathrm{CO}, \mathrm{H}_{2}$, and $\mathrm{PAH}$ emission}

Figure 3 (left) displays a high-resolution CFHT image of the $\mathrm{H}_{2}$ 1-0 S(1) molecular line at $2.12 \mu \mathrm{m}$ (Lemaire et al. 1996), which reveals in detail the filamentary structure of the region. Over-plotted in white and red are the contours of the [O I] $145 \mu \mathrm{m}$ line and one of the excited rotational ${ }^{12} \mathrm{CO}$ line $(J=$ 12-11) measured in the SPIRE data by Köhler et al. (2014). We note that the images are not convolved because the purpose of this panel is to place the emission of [O I] $145 \mu \mathrm{m}$ line and warm $\mathrm{CO}$ relative to the filaments. The right panel of the figure shows the Spitzer $\mathrm{H}_{2}$ 0-0 S(3) $9.7 \mu \mathrm{m}$ map convolved to the $145 \mu \mathrm{m}$ beam, with white [O I] $145 \mu \mathrm{m}$ contours. The $\mathrm{H}_{2}$ 0-0 S(3) transition follows the same distribution as the 1-0 S(1), but because it is a pure rotational line, it peaks slightly behind $\left(\sim 1.9^{\prime \prime}\right)$ the 1-0 S(1) transition. These observations show for the first time that the $[\mathrm{OI}] 145 \mu \mathrm{m}$ emission in NCG 7023 is displaced with respect to the bright filaments that are seen in $\mathrm{H}_{2}$. The reason for this is that since the $\mathrm{H}_{2}$ emission closely traces the UV field, its emission peaks at the edge of the PDR, while the [O I] $145 \mu \mathrm{m}$ is more sensitive to both the gas density and temperature. Köhler et al. (2014) have recently mapped the emission of high-excitation CO lines in the region. This highly excited $\mathrm{CO}$ emission (left panel in Fig. 3) is located at the same position as the $\left[\mathrm{OI}\right.$ ] peak. We note that the ${ }^{12} \mathrm{CO} J=12-11$ line peaks at the same position as the also observed ${ }^{13} \mathrm{CO} J=7-6$ line. Similarly, and although not shown, these condensations are also 

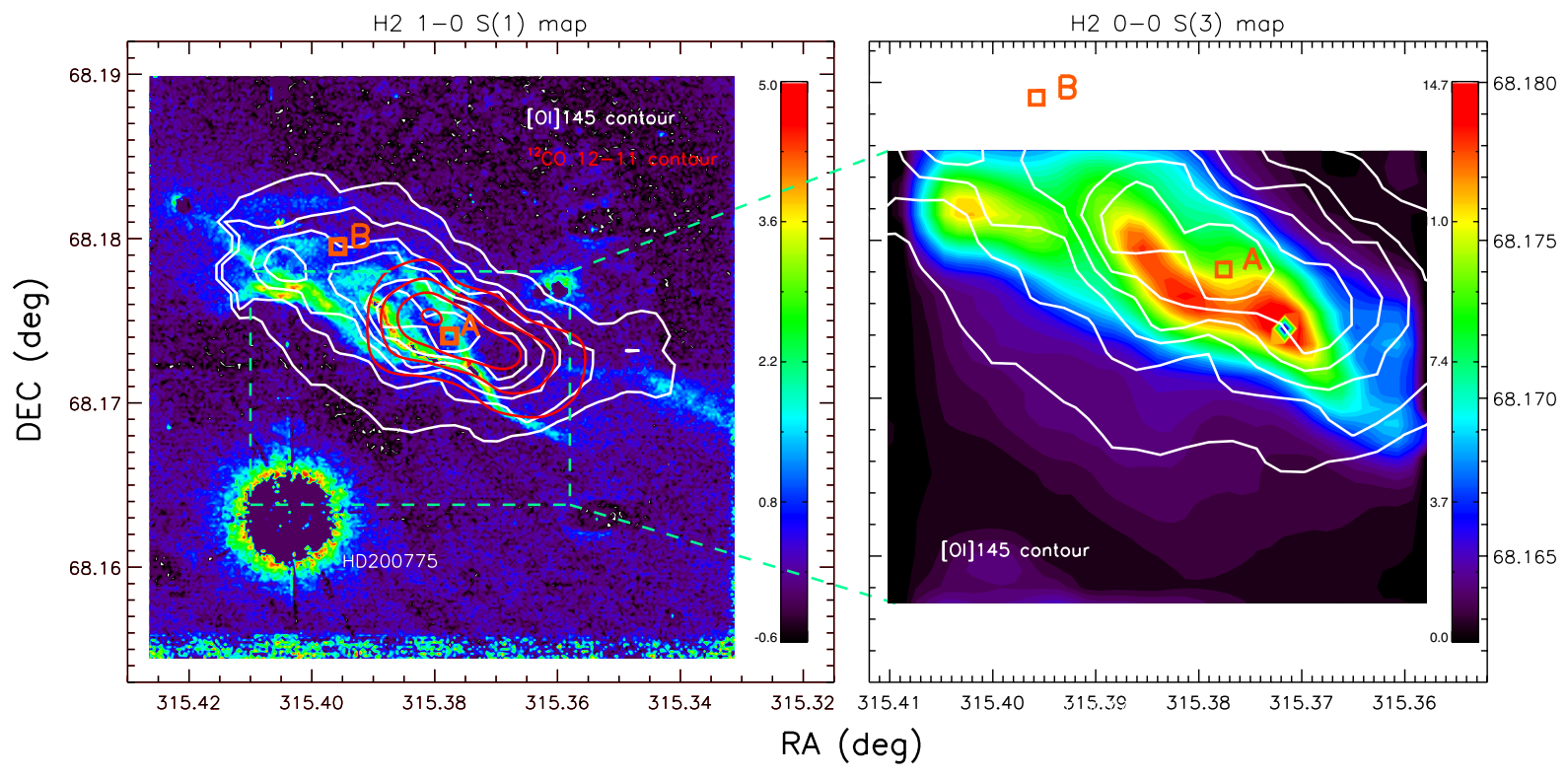

Fig. 3. Left panel: spatial correlation of the $\mathrm{H}_{2}$ 1-0 S(1) emission map at $2.12 \mu \mathrm{m}$ (Lemaire et al. 1996) compared with the [O I] (145 $\left.\mu \mathrm{m}\right)$ and ${ }^{12} \mathrm{CO} J=12-11$ (Köhler et al. 2014) contour maps in white and red. To better illustrate the filaments, these images have not been convolved to the largest beam (in this case, $\mathrm{CO}$ ). The two orange symbols (squares) mark the position where the high-excitation CO lines are detected (A), and a nearby position with similar conditions but without highly excited $\mathrm{CO}$ emission (B). These positions are discussed in Sect. 3.2. Right panel: $\mathrm{H}_{2}$ 0-0 S(3) map at $9.7 \mu \mathrm{m}$ convolved to the [O I] $145 \mu \mathrm{m}$ beam with its contours in white. In both panels the colour bar intensities are in units of $10^{-7} \mathrm{~W} \mathrm{~m}^{-2} \mathrm{sr}^{-1}$, where the four highest contours have values of 5.4, 4.7, 3.9, and 3.1 for [O I] $145 \mu \mathrm{m}$, and of 0.37, 0.3, 0.2, and 0.1 for CO.

detected in Herschel/SPIRE and IRAM submillimetre maps that trace dust emission (Köhler et al. 2014).

It is interesting to explore the origin of this highly excited $\mathrm{CO}$ in relation to the atomic cooling line emission. For this purpose, and to guide the discussion in the next section, we selected two regions: one coinciding with the $\mathrm{CO} J=12-11$ peak (position $A$ ), and a nearby region without high- $J C O$ emission $(B)$. We note that low- $J$ emission is detected in positions $A$ and $B$ (Köhler et al. 2014). Both positions are labelled in Fig. 3. The [O I] $145 \mu \mathrm{m}$ flux and the [O I] $145 \mu \mathrm{m} /[\mathrm{C}$ II] $158 \mu \mathrm{m}$ ratio in position $A$ is 2.2 and 1.7 higher than in position $B$. Although small, these differences are real (larger than the associated uncertainties in the [O I] $145 \mu \mathrm{m}$ flux and ratio), and indicate variations in the physical conditions between the two regions. Such differences can be achieved by changes in the radiation field and/or density of a factor $\sim 3$ in standard PDR grid models (Kaufman et al. 2006). How can such small differences explain the highly excited $\mathrm{CO}$ in position $A$ ? The emission of highly excited $\mathrm{CO}$ can be explained by changes in the gas density if a certain threshold in the conditions is reached. Köhler et al. (2014) derived a density of $5 \times 10^{4}-10^{6} \mathrm{~cm}^{-3}$ and a temperature ranging between $65-130 \mathrm{~K}$ at position $A$, while at position $B$ they found a lower density $\left(10^{4}-10^{5} \mathrm{~cm}^{-3}\right)$ and $T=80-120 \mathrm{~K}$. The critical density of the CO lines $\left(10^{5}-10^{7} \mathrm{~cm}^{-3}\right.$ for $J=4-3$ to 13-12 transitions) is higher than the [OI] lines $\left(\sim 10^{5} \mathrm{~cm}^{-3}\right)$. This means that in the density regime between $10^{4-6} \mathrm{~cm}^{-3}$, the $\mathrm{CO}$ lines are more sensitive than the oxygen lines to an increase in density, which could boost the $\mathrm{CO}$ emission while having little effect on the atomic line emission. It is thus possible that differences in densities in the two positions could explain the highly excited $\mathrm{CO}$ in the clumps.

Because of the low ionisation potential required to ionise carbon $(11.3 \mathrm{eV})$, the [CII] $158 \mu \mathrm{m}$ line can arise from both the neutral (i.e. PDR) and the ionised medium. To validate its use as a star formation indicator, it is interesting to compare the $[\mathrm{CII}]$ emission with other known tracers of the star

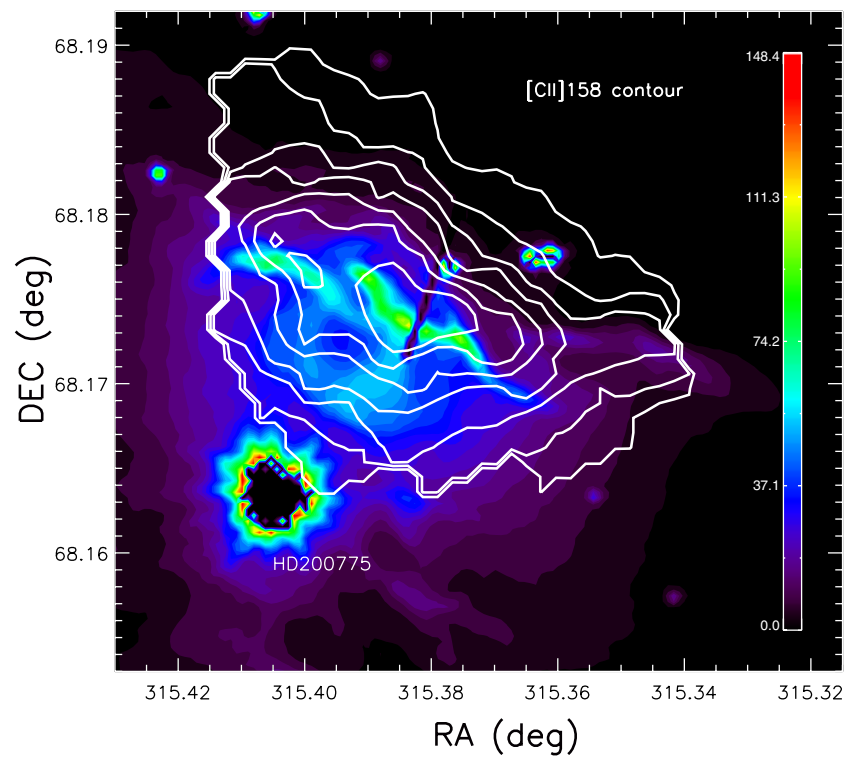

Fig. 4. Comparison of the PAH morphology as traced by the IRAC $3.6 \mu \mathrm{m}$ band intensity in MJy/sr (taken from the Spitzer archive), with [C II] $158 \mu \mathrm{m}$ in white contours. In decreasing order the contour levels are at 10.1, 9.0, 7.8, 6.7, 5.6, 4.5, 3.4, and $2.2 \times 10^{-7} \mathrm{~W} \mathrm{~m}^{-2} \mathrm{sr}^{-1}$.

formation process, such as PAHs (Peeters et al. 2004). In Fig. 4 we make such a comparison, where we plot the PAH emission traced by the Spitzer/IRAC $3.6 \mu \mathrm{m}$ band with the $\left[\mathrm{CII}_{15}\right] \mu \mathrm{m}$ contour map. Although the [C II] $158 \mu \mathrm{m}$ line includes emission from different velocity components, the [C II] line follows the PAH emission reasonably well, with the peak of the [C II] $158 \mu \mathrm{m}$ emission (heart-shaped) coinciding with the PAH filament. Regardless of which regions contribute to the [C II] $158 \mu \mathrm{m}$ emission, they must be (mostly) PDR dominated since it traces the PAH emission. 

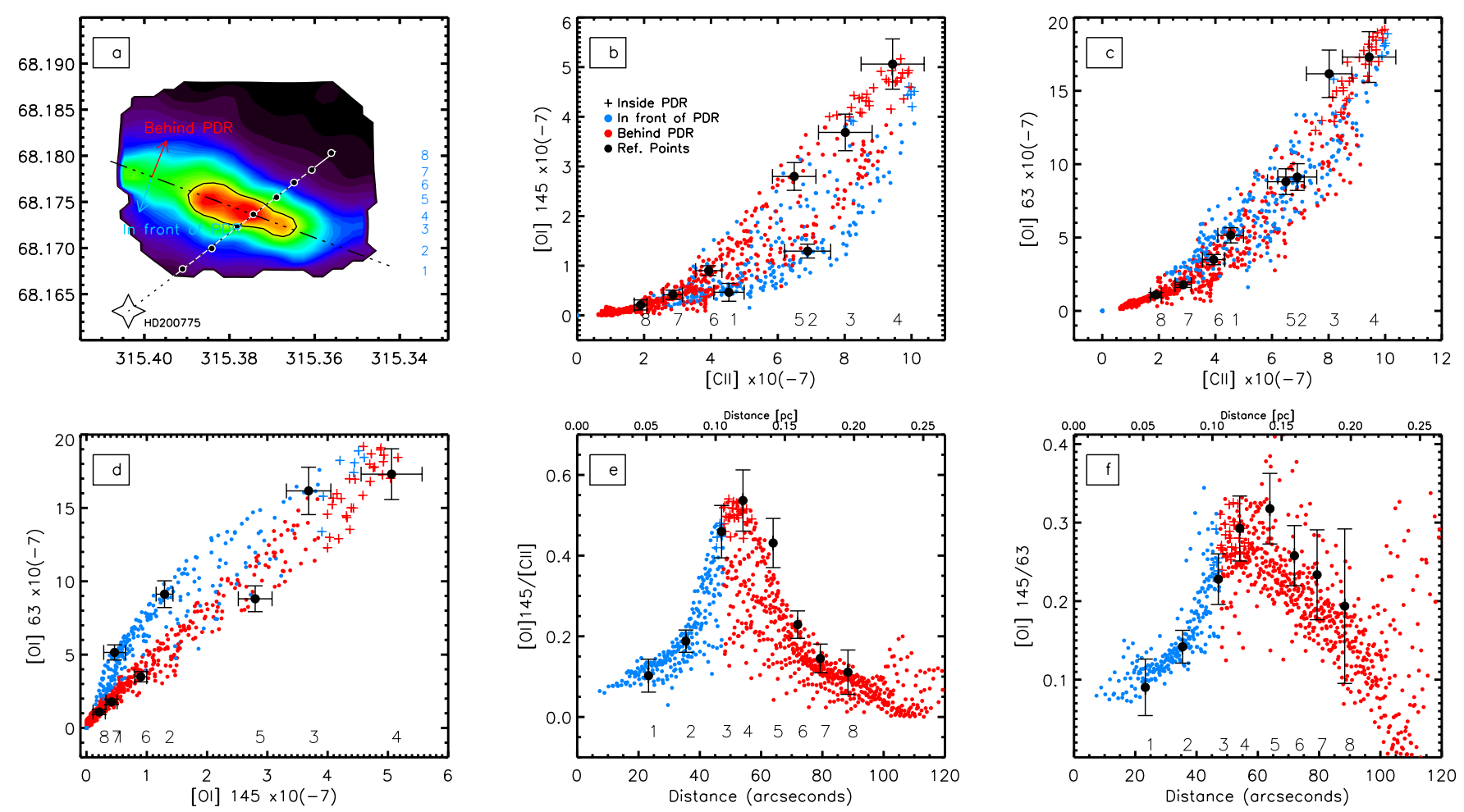

Fig. 5. Intensity plots for different combinations of line fluxes (in $10^{-7} \mathrm{~W} \mathrm{~m}^{-2} \mathrm{sr}^{-1}$ ) and line ratios relative to other lines or distance to HD 200775 . All fluxes have been convolved to the largest beam (11"). The different regions are indicated in the convolved [O I] $145 \mu \mathrm{m}$ map in the first panel and are labelled in the second, where blue represents points in front of the PDR and red those behind it. The peak emission in the PDR is delineated by the black curve in the first panel (those with [O I] $145 \mu \mathrm{m}$ fluxes higher than $75 \%$ of the peak intensity of the line) and are plotted as plus symbols in the subsequent panels. The reference points for the adopted cut are numbered and shown in the first panel (Sect. 5), and have been plotted as black dots with error bars in subsequent panels to give an indication of the uncertainties.

\subsection{Line correlations and ratios}

Different lines and line ratios have been plotted against each other as a function of the distance to the ionising stars HD 20077 in Fig. 5. Panel a in this figure highlights the different regions we consider. These include regions in front of and behind the PDR, which in subsequent panels are shown as blue and red dots. In the figure the plus symbols are PDR points with a flux higher than $75 \%$ of the [OI] $145 \mu \mathrm{m}$ peak emission and are delineated by the black contour. Also shown are selected points from an adopted cut across the PDR starting from HD 200775 (labeled numbers). The latter are shown with their error bars to illustrate the flux uncertainty in different regions of the map. The variation in the flux intensities across the PDR of the [C II] $158 \mu \mathrm{m}$, and [O I]63 and $145 \mu \mathrm{m}$ lines is larger than a factor 10, which is higher than those observed in the Orion Bar (factor $<7$ ) for the same lines (Bernard-Salas et al. 2012).

Panels $b$ and $c$ show the relation between [C II] and [O I]63 and $145 \mu \mathrm{m}$ lines. There is a broad correlation in front of and after the PDR in both plots. The likely reason for the broad relation is the mixture in the line of sight of the different components that give rise to the $[\mathrm{C} \mathrm{II}] 158 \mu \mathrm{m}$ line (see Sect. 3). The relation in panel $\mathrm{c}$ is somewhat narrower than in panel $\mathrm{b}$. This could be attributed to the fact that the emission of the [O I] $63 \mu \mathrm{m}$ line, which is optically thicker than the [O I] $145 \mu \mathrm{m}$ linw, is also extended and follows the [C II] $158 \mu \mathrm{m}$ emission more closely.

Panel d shows a linear correlation between the [OI]63 and $145 \mu \mathrm{m}$ lines behind the PDR, and a more parabolic relationship in front of it. In front of the PDR the gas is warmer and the optical-depth effects become more important. With an
Einstein coefficient five times higher, the [O I]63 $\mu \mathrm{m}$ line will be more self-absorbed than the [O I] $145 \mu \mathrm{m}$ line, and its overall emission will be more extended. Therefore, in that region $[\mathrm{O}$ I] $145 \mu \mathrm{m}$ increases more than the [O I] $63 \mu \mathrm{m}$ line does.

In the last two panels in the figure (e and $\mathrm{f}$ ), the [O I] $145 \mu \mathrm{m} /[\mathrm{C}$ II $] 158 \mu \mathrm{m}$ and [O I] $145 \mu \mathrm{m} /[\mathrm{O} \mathrm{I}] 63 \mu \mathrm{m}$ ratios are plotted against the distance to HD 200775. Closer to the PDR, the density increases and both line ratios increase rapidly, peaking at around $45^{\prime \prime}$. Behind the PDR, the temperature drops and both line ratios decrease. However, and because of the multicomponent $[\mathrm{C}$ II $] 158 \mu \mathrm{m}$ emission, there is a broader relation in the ratio.

\section{Cooling budget}

The mid- and far-IR regions are home to the most important cooling lines in the PDR. Our Herschel observations, together with Spitzer data, have enabled us to estimate the relative importance of these species to the cooling budget. We have thus derived the contribution of the $[\mathrm{CII}]$ and $[\mathrm{OI}]$ cooling lines in the PDR, as well as other relevant atomic and molecular lines in the mid- and far-IR, including $\mathrm{CO}, \mathrm{H}_{2}$, and $\mathrm{C}^{0} . \mathrm{H}_{2}$ and $\mathrm{CO}$ are the most abundance molecular species in the interstellar medium (ISM) and also contribute to the cooling in PDRs (Hollenbach \& Tielens 1999). To calculate the budget of these species, we considered the $0-0 \mathrm{~S}(1), \mathrm{S}(2)$, and $\mathrm{S}(3)$ rotational lines of $\mathrm{H}_{2}$ at 17.0, 12.3, and $9.7 \mu \mathrm{m}$ (Spitzer/IRS), and $\mathrm{CO} 4 \leq J_{\mathrm{u}} \leq 13$ transitions (SPIRE). In the Orion Bar, $\mathrm{C}^{0}, \mathrm{CH}^{+}$and $\mathrm{H}_{2} \mathrm{O}$ lines are detected in the PACS and SPIRE spectra and contribute $\sim 1 \%$ of the total cooling budget 


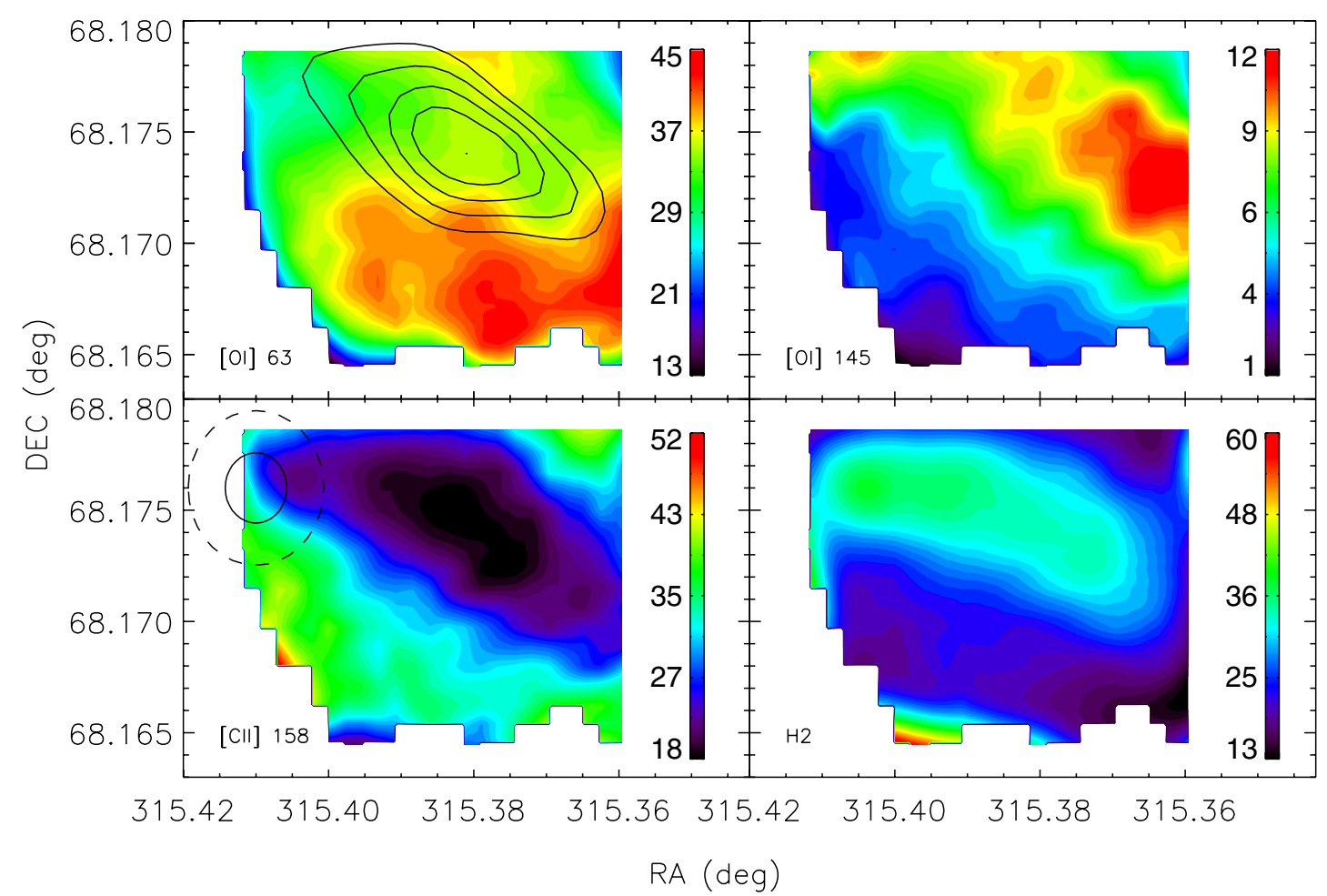

Fig. 6. Relative cooling budget of the [O I $] 63$ and $145 \mu \mathrm{m}$, [C II] $158 \mu \mathrm{m}$ lines, and the $0-0 \mathrm{~S}(1), \mathrm{S}(2), \mathrm{S}(3) \mathrm{H}_{2}$ rotational transitions and $v=1-0 \mathrm{~S}(1)$ in percentage (\%). Adding the contribution of all four species for a given point in the map amounts to $100 \%$. Other species that can contribute to the cooling are ignored in this figure (see Sect. 4). In the first panel we show a contour of the PDR as traced by the [O I] $63 \mu \mathrm{m}$ emission to guide the eye. The images have been convolved to the largest of the beam sizes of these species, in this case the [C II] $158 \mu \mathrm{m}$ line, shown as a solid circle in the [CII] panel. In addition, and for comparison, we also show the largest $\mathrm{CO}$ beam size $\left(25^{\prime \prime}\right)$ used to calculate the cooling budget in Table 1 as a dashed circle.

Table 1. Cooling line emission as a percentage at the PDR position and convolving the emission of all species to a $25^{\prime \prime}$ beam.

\begin{tabular}{lcc}
\hline \hline & NGC 7023 & ${\text { Orion } \text { Bar }^{\dagger}}^{-}$ \\
\hline$[\mathrm{C}$ II $] 158 \mu \mathrm{m}$ & 19 & 8 \\
{$[\mathrm{O} \mathrm{I}] 63 \mu \mathrm{m}$} & 33 & 72 \\
{$[\mathrm{O} \mathrm{I}] 145 \mu \mathrm{m}$} & 9 & 10 \\
{$[\mathrm{C} \mathrm{II}]+[\mathrm{O} \mathrm{I}]$} & 61 & 90 \\
$\mathrm{H}_{2}$ & 35 & 5 \\
$\mathrm{CO}$ & 4 & 5 \\
$\mathrm{C}^{0}, \mathrm{H}_{2} \mathrm{O}, \mathrm{CH}^{+}$ & $<1$ & $<1$ \\
\hline
\end{tabular}

Notes. ${ }^{(\dagger)}$ From Bernard-Salas et al. (2012).

(Habart et al. 2010; Bernard-Salas et al. 2012). This is a small factor compared with the total cooling budget in the Orion Bar, but we also explored the contribution of these lines in NGC 7023. We detected the 370 and $609 \mu \mathrm{m}$ lines of $\mathrm{C}^{0}$ in the SPIRE spectrum (Köhler et al. 2014). We did not detect $\mathrm{CH}^{+}$ and $\mathrm{H}_{2} \mathrm{O}$ transition in the SPIRE spectrum of NGC7023 and therefore derived upper limits for their FIR transitions to estimate their contribution. For this comparison we chose the position of the [OI] emission peak in the PDR (red knots in Fig. 2) and convolved all the line emission to the largest beam, in this case, the SPIRE CO emission (25"). In Table 1 we quote these values together with those of the Orion Bar (Bernard-Salas et al. 2012), which is usually adopted as the prototypical PDR.

Table 1 shows that in the NW PDR of NGC 7023 the cooling budget is dominated by the $[\mathrm{C} \mathrm{II}]+[\mathrm{O} \mathrm{I}](61 \%)$, followed by $\mathrm{H}_{2}$ with $35 \%, \mathrm{CO}(4 \%)$, and $\mathrm{C}^{0}$ contributing less than $1 \%$. The uncertainty in these percentages is around $20 \%$ of each value. The main contributors to the cooling budget are [O I] $63 \mu \mathrm{m}$ and $\mathrm{H}_{2}$. The overall result is similar to that found in the Orion Bar, where the atomic lines dominate the cooling line emission, with [OI]63 $\mu \mathrm{m}$ being the strongest. However, the main difference is the reduced role of $\mathrm{H}_{2}$ in the Orion Bar $(5 \%)$ when compared with NGC 7023. As part of the same program, we studied nine other nearby PDRs and found that like for NGC 7023, $\mathrm{H}_{2}$ contributes to about $30 \%$ (Bernard-Salas et al. in prep.).

In Fig. 6 we show the relative contribution by percentage of the [O I] 63 and $145 \mu \mathrm{m}$, and [C II] $158 \mu \mathrm{m}$ lines, along with that of $\mathrm{H}_{2}$. For $\mathrm{H}_{2}$ we considered the $0-0$ rotational transitions $\mathrm{S}(1)$, $\mathrm{S}(2)$, and $\mathrm{S}(3)$ and the vibrational line $v=1-0 \mathrm{~S}(1)$. All maps were convolved to the $[\mathrm{C}$ II $] 158 \mu \mathrm{m}$ beam. Thus, for a given point in the map, the total cooling of these four species amounts to $100 \%$. Therefore this figure shows which species dominates the cooling in different regions. We ignored the contribution of $\mathrm{C}^{0}, \mathrm{H}_{2} \mathrm{O}$, and $\mathrm{CH}^{+}$, but as we can see from Table 1, their contribution is already negligible in the PDR, where we would expect their contribution to be strongest. If we first look at each map individually, we see that [O I] $63 \mu \mathrm{m}$ contributes more in front of the PDR, and [C II] $158 \mu \mathrm{m}$ outside it (in front and behind). [OI] $145 \mu \mathrm{m}$ and $\mathrm{H}_{2}$ contribute more in the PDR, but with [O I] $145 \mu \mathrm{m}$ contribution being higher in the south-east region (lower right panel). The [O I] $145 \mu \mathrm{m}$ line is more sensitive to the gas density than the $\mathrm{H}_{2}$ lines and thus contributes more where the clumps are located. By reading the percentage from the colour bars, we can also compare the relative contributions. In general, [O I] $63 \mu \mathrm{m}$ is the dominant contributor over the entire region $(>30 \%)$. However, $[\mathrm{C}$ II $] 158 \mu \mathrm{m}$ is a strong coolant outside of the PDR as well (contributing above $30 \%$ ), 

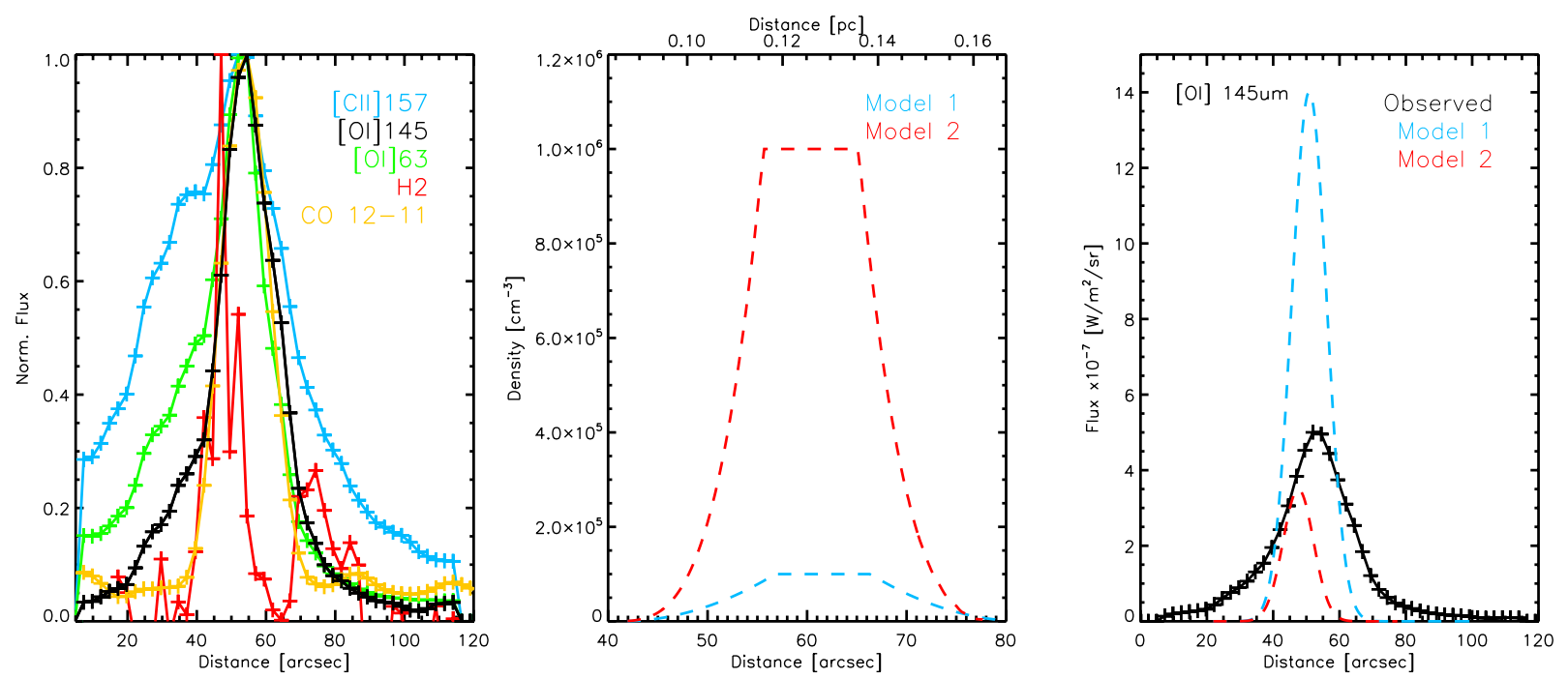

Fig. 7. Left panel: observed non-convolved profile of the atomic and molecular lines as a function of the distance to HD 200775 along the cut shown in Fig. 5 and described in Sect. 4. Middle panel: density profiles at two different maximum densities used by Köhler et al. (2014) in their analysis of the molecular emission. Model 1 has a peak gas density of $10^{5}$, model 2 a density of $10^{6} \mathrm{~cm}^{-3}$. Right panel: observed profile emission of the [O I] $145 \mu \mathrm{m}$ line compared with the convolved predicted emission by Meudon PDR models.

as is $\mathrm{H}_{2}$ in the PDR (>35\%). On the other hand, the contribution of [OI] $145 \mu \mathrm{m}$ does not exceed $12 \%$ in the PDR and is lower than $5 \%$ in the rest of the region. This figure delineates the importance of $[\mathrm{O} \mathrm{I}] 63 \mu \mathrm{m},[\mathrm{C} \mathrm{II}] 158 \mu \mathrm{m}$, and $\mathrm{H}_{2}$ as cooling agents as a function of the region.

\section{Modelling}

In this section our goal is to explore whether previously reported parameters that were used to model the dust emission in the PDR (density profile, geometrical parameters) and CO lines (gas physical conditions) can also reproduce the [O I]145 $\mu \mathrm{m}$ emission. This is important in our quest to find a model that can self-consistently reproduce the dust, molecular, and atomic PDR components. Using Herschel photometry and spectroscopy, the dust emission and molecular content (CO) of the NW PDR has recently been modelled by Arab et al. (2012) and Köhler et al. (2014). In our observations the [C II] $158 \mu \mathrm{m}$ line is a mixture of many components, the emission of the [O I] $63 \mu \mathrm{m}$ line is optically thick, and the [N II] $122 \mu \mathrm{m}$ was not detected. Attempting to model the atomic component from our observations will therefore serve to provide basic physical conditions of the PDR based on fitting the [OI] $145 \mu \mathrm{m}$ line alone. This is why we prefer instead to test whether previously and more detailed modelling studies can also explain the [O I] $145 \mu \mathrm{m}$ emission.

To model the line emission we selected a cut originating from the ionising stars that intersected the NW PDR at a point of peak dust emission at $250 \mu \mathrm{m}$. This is the same cut as was used in (Köhler et al. 2014) for the molecular line and dust emission. The observed emission profiles for the three lines, together with that of the $\mathrm{H}_{2} 1-0 \mathrm{~S}(1)$ and $\mathrm{CO} J=12-11$ lines, are shown in Fig. 7 (left panel). These profiles are shown at their natural resolution to maximise the spatial information we can obtain from them. [O I]63 and $145 \mu \mathrm{m},[\mathrm{C} \mathrm{II}] 158 \mu \mathrm{m}$, and CO $J=12-11$ peak at the same position. Because it has the highest spatial resolution, the $\mathrm{H}_{2}$ profile resolves two filaments (see Fig. 3), with the densest filament peaking immediately in front of the fine-structure and $\mathrm{CO}$ lines relative to the external illumination. The $[\mathrm{C}$ II $] 158 \mu \mathrm{m}$ line has the broadest profile, followed by $[\mathrm{O}$ I $] 63 \mu \mathrm{m}$.
We modelled the emission of the [O I] $145 \mu \mathrm{m}$ line with the Meudon PDR radiative transfer code (version 1.4.4, Le Petit et al. 2006; Le Bourlot et al. 2012). This code assumes a planeparallel slab of gas and dust that is illuminated by an incident radiation field and iteratively resolves the radiative transfer and thermal and chemical balance to compute the atomic and molecular emission in the cloud. As in the papers listed above, we assumed an incident radiation field of $G \sim 2600$ on the NW PDR at $42^{\prime \prime}$ from the star (Chokshi et al. 1988; Pilleri et al. 2012). The RADEX analysis of the CO lines (Köhler et al. 2014) and the modelling of the dust emission are compatible with two maximum gas densities of $10^{5}$ and $10^{6} \mathrm{~cm}^{-3}$. We therefore considered both possibilities for our comparison. These studies derived a PDR length of $0.2 \mathrm{pc}$ for a gas density $10^{5} \mathrm{~cm}^{-3}$ and $0.03 \mathrm{pc}$ for a density of $10^{6} \mathrm{~cm}^{-3}$, giving both a total column density of about $10^{22} \mathrm{~cm}^{-2}$, and we adopted these values in our study. We assumed the density profile derived from the dust analysis and adjusted to start at $0.088 \mathrm{pc}\left(42^{\prime \prime}\right)$ from HD 200775. The density profiles for the two gas densities we considered are shown in the middle panel of Fig. 7. We hereafter refer to these models as model $1\left(n=10^{5} \mathrm{~cm}^{-3}\right)$ and model $2\left(n=10^{6} \mathrm{~cm}^{-3}\right)$.

Before we proceed, it is interesting to investigate whether the depth of the PDR deduced from the previous studies (Arab et al. 2012; Köhler et al. 2014) is compatible with the width and length of the PDR we derived from the observations (spatial extent of the [O I] $145 \mu \mathrm{m}$ line). In the middle top panel of Fig. 2, the PDR has an approximate width of $20^{\prime \prime}$ and a length of $60^{\prime \prime}$ (as traced by the green contours in the figure), which corresponds to 0.04 and 0.12 pc. From model 1 we find that the depth is about twice the observed length, resulting in a flat extended geometry. For model 2 the depth compares very well to the projected width, resulting in a cylindric geometry.

The predicted [OI]145 $\mu \mathrm{m}$ intensities for models 1 and 2 are shown in the right-hand panel of Fig. 7, where the line emissivities have been convolved to the PACS beam at $145 \mu \mathrm{m}$. For this comparison we considered the conditions of the models in the region where the predicted unconvolved flux is greater than half the maximum flux $\left(48^{\prime \prime}-52^{\prime \prime}\right.$ from the binary system). In this region model 1 gives $n=10^{4}-8 \times 10^{4} \mathrm{~cm}^{-3}$ and $T=90-530 \mathrm{~K}$. Model 2 gives $n=2 \times 10^{4}-2 \times 10^{5} \mathrm{~cm}^{-3}$ and 
$T=90-660 \mathrm{~K}$. In both models most of the emission comes from a region with a visual extinction $A_{V}=0.07-1.2$. With a $10 \%$ uncertainty in the observed line, none of the models reproduces the [O I] $145 \mu \mathrm{m}$ peak intensity. Note that we did not attempt to fit the line, but this additional constraint may serve to discriminate between the two models. Model 1 predicts an intensity nearly three times higher than the observations, while model 2 underestimates the emission by just 30\%. The maximum gas temperature in both models is higher than the gas temperature derived by Köhler et al. (2014) when modelling the CO $J=13-12$ to $J=6-5$ lines with $\operatorname{RADEX}(140 \mathrm{~K})$. The higher temperature we find is expected since models predict that the $[\mathrm{O}$ I] $145 \mu \mathrm{m}$ line originates from a slightly warmer layer than that of the intermediate $J \mathrm{CO}$ excited lines.

The fact that we find a model that can reproduce the dust emission and also roughly matches the spatial profile of the [O I] $145 \mu \mathrm{m}$ line is an encouraging step towards a self-consistent model that explains both the gas and dust. However, while model 2 fails to exactly reproduce the peak and width of the [OI] emission, we must bear in mind that we are assuming a simple model with a simple geometry. The $\mathrm{H}_{2}$ observations reveal a more complex structure with several filaments. The density profile we used also reflects a smooth PDR, while our own observations clearly reveal a dense structure inside of the PDR. Our cut is also arbitrary. These complex structures will affect the $[\mathrm{OI}]$ emission profile (width, peak position). Given these caveats, it is therefore expected that we do not have a perfect fit. To explain the intensity peak of the excited $\mathrm{CO}$ emission, a steeper density gradient at the PDR edge over a small scale is needed to have a large amount of both warm and dense gas. The $\mathrm{CO}$ lines can be reproduced using a high-pressure model $\left(P=10^{8} \mathrm{~K} \mathrm{~cm}^{-3}\right)$ and relatively small PDR width and depth of 2-3" (Joblin et al., in prep.). Such a model can also predict the maximum peak intensity of the [O I] $145 \mu \mathrm{m}$ line. A lower pressure is needed, however, to reproduce the full extent of the PDR that we have presented in Fig. 2.

Finally, and since the Meudon code does not takes into account the ionised region, we modelled the emission of the [N II] 122 and $205 \mu \mathrm{m}$ lines using the radiative transfer code Cloudy (Ferland et al. 1998). We used the same density profile, peak density, and PDR length as in $\operatorname{model}^{3} 2$. For the radiation field, we assumed a Kurucz spectrum (Kurucz 1993) with $T_{\text {eff }}=15000 \mathrm{~K}$ corresponding to the spectral type (B3V-B5V) of the illuminating binary star (Alecian et al. 2008), but attenuated to have an incident radiation field of $G_{0} \sim 2600$ at the edge of the PDR (Pilleri et al. 2012). While there is a cavity, the ionising sources are young and probably have some material around the star. This attenuation of the radiation field is probably caused by the envelope of the star and the dilution effect. We also adopted ISM abundances (Savage \& Sembach 1996; Meyer et al. 1997, 1998). There is a cavity between the star and the PDR. The density profile is adjusted to start at $0.023 \mathrm{pc}\left(11^{\prime \prime}\right)$ from HD 200775. The intensity of the nitrogen lines at 122 and $205 \mu \mathrm{m}$ are sensitive to the electron density in the ionised region, and on the incident radiation field, but we did not detect either line. The upper limits we derived for these lines $\left(1.1 \times 10^{-7}\right.$ and $\left.10^{-9} \mathrm{~W} \mathrm{~m}^{-2} \mathrm{sr}^{-1}\right)$ are compatible with two scenarios for the cavity, one with an ionised region and a density $n<100 \mathrm{~cm}^{-3}$, and another one without an ionised region and a density of $500 \mathrm{~cm}^{-3}$.

\footnotetext{
3 Using the parameters of model 1 does not change the conclusion.
}

\section{Summary and conclusions}

We have presented Herschel/PACS spatially resolved observations of the [C II] $158 \mu \mathrm{m},[\mathrm{OI}] 63 \mu \mathrm{m}$ and $145 \mu \mathrm{m}$ lines of the NW PDR in NGC 7023. This has enabled us to study the emission of these cooling lines in relation to the morphology of the region. We summarise the main findings here:

1. The emission of the atomic cooling lines trace the cloud surface that is directly illuminated by the binary system HD 200775. Here the gas is warmer, and these lines are associated with the filamentary structure revealed in higherresolution $\mathrm{H}_{2}$ and $\mathrm{PAH}$ maps. In the PDR, the peak of cooling line emission corresponds to condensations that are seen from high-level rotational CO lines and Herschel and submillimetre emission of dust.

2. By comparing the role of different coolants, we found that the $[\mathrm{OI}] 63$ and $145 \mu \mathrm{m}$ and $[\mathrm{C}$ II] $158 \mu \mathrm{m}$ lines account for $61 \%$ of the cooling in the PDR, with [OI]63 $\mu \mathrm{m}$ contributing to $33 \%$ of the emission. $\mathrm{H}_{2}$ also contributes significantly with $35 \%, \mathrm{CO}$ with $4 \%$, and other atoms and molecules $\left(\mathrm{C}^{0}, \mathrm{H}_{2} \mathrm{O}, \mathrm{CH}^{+}\right)$contribute less than one percent. The relative contribution of the main cooling agents over the region shows that while the $[\mathrm{OI}] 63 \mu \mathrm{m}$ dominates the cooling, $\mathrm{H}_{2}$ also contributes significantly in the PDR, and [C II] $158 \mu \mathrm{m}$ is also important in front of and behind the PDR. This highlights the importance of [O I] $63 \mu \mathrm{m}$ and $\mathrm{H}_{2}$ as a coolant in the PDR and of [C II] $158 \mu \mathrm{m}$ in lower excited regions. It is interesting to note that while in the Orion Bar the atomic lines dominate the cooling, $\mathrm{H}_{2}$ contributes to only $5 \%$.

3. The [O I]63 and $145 \mu \mathrm{m}$ maps spatially resolve these condensation into two distinct structures. Furthermore, Herschel's high angular resolution shows that the [O I] $145 \mu \mathrm{m}$ emission peaks slightly farther away from the ionising stars than the $\mathrm{H}_{2}$ emission, which traces the edge of the PDR. This is expected since [O I] $145 \mu \mathrm{m}$ is more sensitive to the gas density.

4. [O I]63 and $145 \mu \mathrm{m}$ and [C II] $158 \mu \mathrm{m}$ peak at the same position. The $[\mathrm{C}$ II $] 158 \mu \mathrm{m}$ line is more extended than the [O I]63 and $145 \mu \mathrm{m}$ lines because [C II] $158 \mu \mathrm{m}$ is easily excited and presents a more complex structure. This is consistent with HIFI observations of the region by (Joblin et al. 2010), which revealed different velocity components for the $[\mathrm{C} \mathrm{II}] 158 \mu \mathrm{m}$ emission, indicating that different regions contribute to the emission of this line.

5. The emission of highly excited ${ }^{12} \mathrm{CO} J=12-11$ correlates with the peak of emission of the cooling lines. Emission from the atomic cooling lines and line ratios at this position differs by a factor of two compared with the conditions of a nearby position without $\mathrm{CO} J=12-11$ emission. This difference in the cooling line emission indicates a change in the physical conditions (density varying from $10^{4}$ to $10^{5-6} \mathrm{~cm}^{-3}$ ). In this density range, the $\mathrm{CO}$ lines are more sensitive than the atomic lines, which might explain the warm $\mathrm{CO}$ in this region.

6. Using a density profile derived to reproduce the dust emission and physical parameters from the analysis of CO lines, we have modelled the emission of the [OI]145 $\mu \mathrm{m}$ line using two different peak gas densities with the Meudon code. The best model predicts the [O I] $145 \mu \mathrm{m}$ emission to within $30 \%$ of the intensity at the peak of the emission, occurring at $A_{\mathrm{V}}=0.07-1.2$. In this region the conditions are $n=1.7 \times 10^{4}-1.8 \times 10^{5} \mathrm{~cm}^{-3}$ and $T=90-660 \mathrm{~K}$. The PDR depth along the line of sight needed to reproduce the 
absolute [O I] $145 \mu \mathrm{m}$ intensity is $0.03 \mathrm{pc}$, which is similar to the observed width of the PDR in our map.

7. We did not detect the ionised line of [N II] at $122 \mu \mathrm{m}$. Our Cloudy model indicates that the ionised shell may have a low density, in which case the [N II] $122 \mu \mathrm{m}$ line is too weak to be detected in our observations. Upper limits to this line and the [N II] at $205 \mu \mathrm{m}$ line are consistent with two scenarios for the cavity, one with an ionised region and a density $n<$ $100 \mathrm{~cm}^{-3}$, and one without an ionised region and a density of $500 \mathrm{~cm}^{-3}$. This is supported by HIFI observations, which reveal that the $[\mathrm{N} \mathrm{II}]$ at $205 \mu \mathrm{m}$ line only emits in a shell close to the star, and indicates that the cavity between the star and the PDR is mostly filled with non-ionised gas.

The morphology of the [O I]63 and $145 \mu \mathrm{m}$ and [C II] $158 \mu \mathrm{m}$ lines gives an unprecedented view of the variation of the physical conditions, energetics, and cooling across the NW PDR. The clumps of emission we observe provide a means to understanding how stellar radiation interacts with the gas, and in the future it will be interesting to model whether such clumps will be photo-evaporated or form stars. Given the complexity of the region and that we have assumed a simple planar model to reproduce the $[\mathrm{O} \mathrm{I}] 145 \mu \mathrm{m}$ using parameters derived from modelling the dust and $\mathrm{CO}$ emission, this study offers an encouraging step forward in producing a self-consistent model that can explain the atomic, molecular, and dust emission. Moreover, spatially resolved studies like this can help us calibrate the use of the [C II] $158 \mu \mathrm{m}$ and [O I] lines to trace star formation (Stacey et al. 1991; Meijerink et al. 2007) and understand the origin of the socalled [CII] deficit (Luhman et al. 2003) in most luminous galaxies. Because of its brightness, the [C II] $158 \mu \mathrm{m}$ line provides an unmatched opportunity for redshift determinations and source diagnostics of far distant systems, especially with ALMA.

Acknowledgements. We thank an anonymous referee for useful comments and suggestions. J.B.S. wishes to acknowledge the support from a Marie Curie Intra-European Fellowship within the 7th European Community Framework Program under project number 272820. M.H.D.v.d.W. is supported by CSA and NSERC. We thank C. Joblin for useful comments and discussion. HCSS, HSpot, and HIPE are joint developments by the Herschel Science Ground Segment Consortium, consisting of ESA, the NASA Herschel Science Center, and the HIFI, PACS and SPIRE consortia. PACS has been developed by a consortium of institutes led by MPE (Germany) and including UVIE (Austria); KU Leuven, CSL, IMEC (Belgium); CEA, LAM (France); MPIA (Germany); INAF-IFSI/OAA/OAP/OAT, LENS, SISSA (Italy); IAC (Spain). This development has been supported by the funding agencies BMVIT (Austria), ESAPRODEX (Belgium), CEA/CNES (France), DLR (Germany), ASI/INAF (Italy), and CICYT/MCYT (Spain).

\section{References}

Abergel, A., Arab, H., Compiegne, M., et al. 2010, A\&A, 518, L96 Alecian, E., Catala, C., Wade, G. A., et al. 2008, MNRAS, 385, 391 Arab, H., Abergel, A., Habart, E., et al. 2012, A\&A, 541, A19 Benisty, M., Perraut, K., Mourard, D., et al. 2013, A\&A, 555, A113 Bernard-Salas, J., Habart, E., Arab, H., et al. 2012, A\&A, 538, A37 Caux, E., Ceccarelli, C., Castets, A., et al. 1999, A\&A, 347, L1

Chokshi, A., Tielens, A. G. G. M., Werner, M. W., \& Castelaz, M. W. 1988, ApJ, 334,803

Ferland, G. J., Korista, K. T., Verner, D. A., et al. 1998, PASP, 110, 761

Fleming, B., France, K., Lupu, R. E., \& McCandliss, S. R. 2010, ApJ, 725, 159

Fuente, A., \& Martín-Pintado, J. 1997, ApJ, 477, 107

Fuente, A., Martiín-Pintado, J., Cernicharo, J., Brouillet, N., \& Duvert, G. 1992, A\&A, 260, 341

Fuente, A., Martiín-Pintado, J., Neri, R., Rogers, C., \& Moriarty-Schieven, G. 1996, A\&A, 310, 286

Fuente, A., Martín-Pintado, J., Rodríguez-Fernández, N. J. 1999, ApJ, 518, L45

Fuente, A., Martín-Pintado, J., Rodríguez-Fernández, N. J., Cernicharo, J., \& Gerin, M. 2000, A\&A, 354, 1053

Habart, E., Dartois, E., Abergel, A., et al. 2010, A\&A, 518, A116

Habing, H. J. 1968, Bull. Astr. Inst. Netherlands, 19, 421

Hollenbach, D. J., \& Tielens, A. G. G. M. 1999, Rev. Mod. Phys., 71, 173

Joblin, C., Pilleri, P., Montillaud, J., et al. 2010, A\&A, 521, L25

Kaufman, M. J., Wolfire, M. G., \& Hollenbach, D. J. 2006, ApJ, 644, 283

Köhler, M., Habart, E., Arab, H., et al. 2014, A\&A, 569, A109

Kurucz, R. L. 1993, VizieR Online Data Catalog VI/39

Le Bourlot, J., Le Petit, F., Pinto, C., Roueff, E., \& Roy, F. 2012, A\&A, 541, A76

Le Petit, F., Nehmé, C., Le Bourlot, J., \& Roueff, E. 2006, ApJS, 164, 506

Lebouteiller, V., Cormier, D., Madden, S. G., et al. 2012, A\&A, 548, A91

Lemaire, J. L., Field, D., Gerin, M., et al. 1996, A\&A, 308, 895

Luhman, M. L., Satyapal, S., Fischer, J., et al. 2003, ApJ, 594, 758

Meijerink, R., Spaans, M., \& Israel, F. P. 2007, A\&A, 461, 793

Meyer, D. M., Cardelli, J. A., \& Sofia, U. J. 1997, ApJ, 490, 103

Meyer, D. M., Jura, M., \& Cardelli, J. A. 1998, ApJ, 493, 222

Okada, Y., Pilleri, P., Berné, O., et al. 2013, A\&A, 553, A2

Ossenkopf, V., Röllig, M., Kramer, C., et al. 2011, EAS Pub. Ser., 52, 181

Ott, S. 2010, ASP Conf. Ser., 434, 139

Peeters, E., Mattioda, A. L., Hudgins, D. M., \& Allamandola, L. J. 2004, ApJ, 617, 65

Pilbratt, G. J., Riedinger, J. R., Passvogel, T., et al. 2010, A\&A, 518, A2

Pilleri, P., Montillaud, J., Berné, O., \& Joblin, C. 2012, A\&A, 542, A69

Poglitsch, A., Waelkens, C., Geis, N., et al. 2010, A\&A, 518, A2

Savage, B. D., \& Sembach, K. R. 1996, ARA\&A, 34, 279

Sellgren, Werner, M. W., \& Dinerstein, H. L. 1992, ApJ, 400, 238

Sellgren, K., Uchida, K. I., \& Werner, M. W. 2007, ApJ, 659, 1338

Sellgren, K., Werner, M. W., Ingalls, J. G., et al. 2010 ApJ, 722, L54

Stacey, G. J., Geis, N., Genzel, R., et al. 1991, ApJ, 373, 423

van den Ancker, M. E., Thé, P. S., Tjin A Djie, H. R. E., et al. 1997, A\&A, 324, L33

van Leeuwen, F. 2007, A\&A, 474, 653

Werner, M., Roellig, T. L., Low, F. J., et al. 2004, ApJS, 154, 1

Witt, A. N., Gordon, K. D., Vijh, U. P., et al. 2006, ApJ, 636, 303 